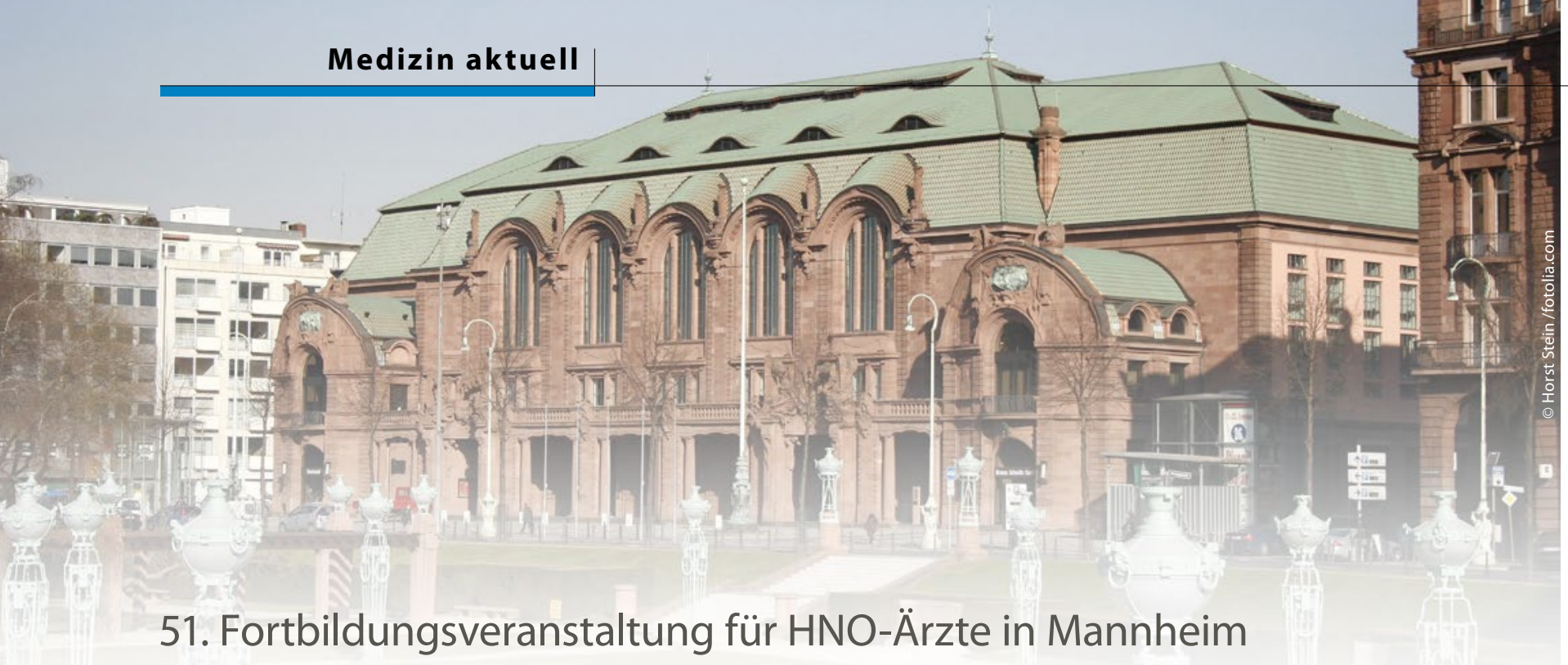

\title{
Kopf-Hals-Tumoren interdisziplinär behandeln
}

\author{
Onkologische Erkrankungen waren einer der Schwerpunkte der diesjährigen Fortbildungsveranstaltung für \\ Hals-Nasen-Ohrenärzte vom 26. bis 28. Oktober in Mannheim. Das vielfältige Programm zeigte: Die optimale \\ Betreuung von Tumorpatienten erfordert interdisziplinäre Zusammenarbeit.
}

\section{Vestibularisschwannom: beobachten, bestrahlen oder operieren?}

$B^{\text {eis }}$ ei Patienten mit Vestibularisschwannom ist der Erhalt oder wenn möglich die Verbesserung der Lebensqualität das vorrangige Ziel der Behandlung. Durch welche Strategie dies am besten gelingt, sollte im individuellen Fall interdisziplinär abgewogen werden, wie Experten im Rahmen eines Rundtischgesprächs anhand zahlreicher Fallbeispiele illustrierten.

Aktuell entwickelte Behandlungspfade [Rosahl S, Bohr C, Lell M, Hamm K, Iro H: Laryngorhinootologie 2017; 96 (S 01): S152-S182] können für die Entscheidungsfindung hilfreich sein, erläuterte Prof. Dr. Steffen Rosahl, Klinik für Neurochirurgie, HELIOS Klinikum Erfurt. Wichtige Kriterien sind neben der Größe des Tumors seine Wachstumsgeschwindigkeit, das Hörvermögen sowie Alter und Vorerkrankungen des Patienten.

Bei kleineren Tumoren (unter $25 \mathrm{~mm}$ Durchmesser), insbesondere bei älteren Patienten, wird in vielen Fällen zunächst der spontane Verlauf unter regelmäßiger klinischer und MRT-Kontrolle beobachtet. Das Wachstumsverhalten des gutartigen Tumors gibt auch prognostische Hinweise auf den Hörerhalt sowie die Wahrscheinlichkeit eines Rezidivwachstums nach unvollständiger Entfernung, die in die weitere Beratung des Patienten mit einfließen.

Alternativ zur Strategie „wait and scan" bietet die Bestrahlung gerade bei kleineren Tumoren und funktionellem Hörvermögen die Möglichkeit, unter Hörerhalt einen Wachstumsstopp des Tumors zu erreichen, sagte Priv.-Doz. Dr. Klaus Hamm, CyberKnife Zentrum Mitteldeutschland im HELIOS Klinikum Erfurt. Versierten Chirurgen gelingt es aber auch, den Tumor unter Erhalt des Hörvermögens vollständig zu entfernen.

Bei Wachstum des Tumors oder Verschlechterung der Beschwerden sollte erneut eine Beratung im interdisziplinären Tumorboard erfolgen, anschließend sollten mit dem Patienten die radiooder mikrochirurgischen Behandlungsoptionen besprochen werden.

Auch bei größeren Tumoren (Durchmesser über $25 \mathrm{~mm}$ ) kann bei Patienten über 70 Jahren eine beobachtende Strategie sinnvoll sein, jüngeren Patienten wird eher zu einer Operation geraten. Bei Hirnstammsymptomatik ist eine weitere Beobachtung allerdings keine sinnvolle Option. Für größere Tumoren wird eine primär radiochirurgische oder stereotaktisch radiotherapeutische Behandlung in der Regel nicht empfohlen. Die Bestrahlungstherapie kann aber bei Rest- oder Rezidivtumoren nach einer Operation oder bei hohem OP-Risiko in Frage kommen.

Der Patient sollte ausführlich über die Vorteile und Risiken der verschiedenen Managementstrategien aufgeklärt werden. Für die Therapieentscheidung spielen auch die Wünsche des Patienten eine gewichtige Rolle, betonte Prof. Dr. Dr. Heinrich Iro, Universitäts-HNO-Klinik Erlangen.

Rundtischgespräch: Interdisziplinäre Behandlung des Vestibularisschwannoms 